\title{
Breach of the Greater Omentum through the Bladder then the Urethra: A Case Report
}

Mourad Badri", Hicham EL Bouhadouti, Ouadii Mouaqit, Karim Ibn Majdoub Hassani, Imane Toughraï, Abdelmalek Oussaden, Khalid Mazaz, Khalid Ait Taleb, El Bachir Benjelloune

Department of Visceral and Endocrinological Surgery, Chu Hassan II, Fes, Morocco

DOI: $10.36347 /$ sjmcr.2020.v08i03.028

| Received: 05.03.2020 | Accepted: 12.03.2020 | Published: 19.03.2020

*Corresponding author: Mourad Badri

Abstract

Case Report

Perforation of the bladder, as a late complication of radiation therapy, is a rare condition, especially on a non-necrotic bladder. We report in this publication, the case of a patient, who 1 and a half year after the end of her irradiation, presented a complication characterized by a bladder perforation with intrusion of the greater omentum through the bladder, along the ureter to the outside, to subsequently necrose and expose the patient to a risk of urinary peritonitis.

Keywords: breach of bladder, great omentum, radiotherapy.

Copyright @ 2020: This is an open-access article distributed under the terms of the Creative Commons Attribution license which permits unrestricted use, distribution, and reproduction in any medium for non-commercial use (NonCommercial, or CC-BY-NC) provided the original author and source are credited.

\section{INTRODUCTION}

Radiation therapy, whether or not combined with chemotherapy, is a treatment of choice that finds its indication in locally advanced cervical cancers or in pre-surgery. The inventory of the complications of this therapy made it possible to classify them as early and late, which can affect all the exposed organs.

\section{Case Report}

A 50-year-old patient, followed in the radiotherapy department for moderately differentiated epidermoid carcinoma of the cervix, for which she received concomitant radio-chemotherapy at a total dose of 70 grays with good progress. Patient lost to follow-up and then readmitted after a year and a half with metrorrhagia and a tumor recurrence that had become advanced, non-surgical. The decision was to put the patient on palliative chemotherapy.

We admitted this same patient to adult emergency for breacking of her great omentum through the urethra via a perforation of her bladder.

The patient was conscious, hemodynamically and respiratory stable; with a pulse at $80 \mathrm{~b} / \mathrm{m}$, a temperature at 38.2 and normo-colored conjunctiva. The clinical examination found hypogastric sensibility and a rest of the abdomen that was flexible. At the pelvic level, there was necrotic fatty tissue through a much-dilated urethra.
On the biological level: hemoglobin was at $8 \mathrm{~g}$ / dl, leukocytes at $12200 / \mathrm{uL}, \mathrm{CRP}$ at $387 \mathrm{mg} / \mathrm{L}$, the rest of the balance was no perturbations.

The patient benefited from an abdominal CT that found out a prolapse through the urethra with predominantly fatty content containing a part of the greater omentum as well as a part of a jejunal loop without no sign of ischemia in the intestine.

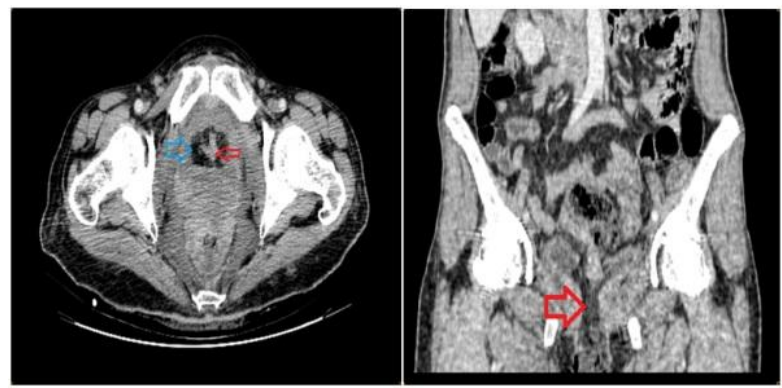

In blue: perforation of the bladder. In red: breach of the great omentum

After pre-anesthetic assessment, we took the patient to the operating room where under general anesthesia, in lithotomy position, we proceeded by a sub umbilical median laparotomy. The exploration revealed an intraperitoneal effusion of about $1.5 \mathrm{~L}$ made of urine with a perforation of $2 \mathrm{~cm}$ from the anterosuperior edge of the bladder through which the large omentum necroses over the entire extent of the prolapsed part. We therefore removed and aspirated the 
effusion fluid, reintroduced the greater omentum intraperitoneally, resected the necrotic portion of the greater omentum and closed the bladder breach. We then did a tightness test, washed and drained the peritoneal cavity by a drain at the level of the pelvis.

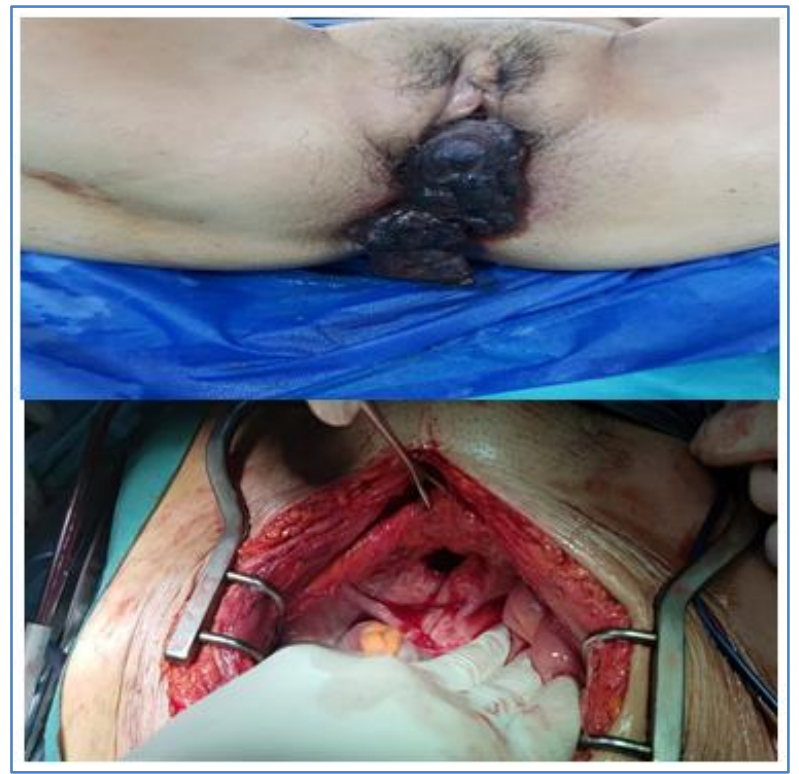

On the top: breach of the great omentum through the urethra On the bottom: perforation of the bladder after removing the great omentum

The postoperative follow-up was simple and the patient discharged after 2 days.

\section{DISCUSSION}

Studies have shown that concomitant radiochemotherapy reduces the risk of recurrence by up to $50 \%$ in patients with advanced cervix cancer, regional spread or high-risk characteristics after hysterectomy. In contrast, studies on neoadjuvant chemotherapy followed by radiotherapy have repeatedly failed to show a survival advantage [1-3].

For stages from IB2 to IV A of epidermoid carcinoma of the cervix, the standard treatment is concomitant radio-chemotherapy. Primary surgery to remove the tumor is not indicated. The irradiation is pelvic +/- lumbar-aortic and spans 5 weeks. Uterovaginal brachytherapy is performed 8 to 10 days after the end of external radiotherapy. The most frequently used chemotherapy is based on platinum salts (5-FU Platinum), administered weekly during radiotherapy, i.e. 5 to 6 courses [4].

The late complications of concomitant radiochemotherapy and more specifically the long-term effect of irradiation of the cervix tumor, deemed nonsurgical, are, according to the editorial of The American Cancer Society, published on January 032020 [5]:

- Vaginal dryness and stricture.

- Rectal stenosis and rectal bleeding.
- Episodes of sub-occlusion or even occlusion by small bowel radiation.

- Chronic radiation cystitis with more or less disabling pollakiuria and bladder instability syndrome, hematuria, vesico-vaginal fistula. Bladder complications are getting more serious by the addition of chemotherapy, compared to patients who receive radiotherapy alone [6].

- Pathological fractures of the pelvic bones.

- Lymphedema due to affection of the lymphatic drainage of the lower limbs.

A classification in 5 grades of the morbidity of radiotherapy at the genitourinary level has been proposed by the Radiation Therapy Oncology Group, member of the National Cancer Institute, as follows [7]:

Grade 0: No symptomatology

Grade 1: Day or night pollakiuria twice as frequent as before treatment. Dysuria and urgency that do not require treatment

Grade 2: Day or night pollakiuria less frequent than hourly. Dysuria, urgency, or bladder spasm requiring a local anesthetic.

Grade 3: Imperious pollakiuria at least every hour. Dysuria, pelvic pain or bladder spasm requiring regular and frequent painkillers. Significant haematuria with or without emission of clots

Grade 4: Hematuria requiring transfusions. Bladder obstruction not secondary to a clot release. Bladder ulceration or necrosis

Grade 5: Toxicity resulting in death of the patient

The breach of the bladder wall, as a complication of radiotherapy, remains a rare event [8], but which remains present in the Asian series, especially Japanese ones, because of the high doses of brachytherapy associated with radiotherapy in the treatment cervical cancer [9].

The case of our patient arouses interest mainly because of its rarity but also of the attitude of the great omentum which crossed the perforation to then emerge through the urethra which it dilated. This exposed the patient, among other things, to a risk of urinary peritonitis.

\section{Conclusion}

Finally, since radiotherapy is widely used for cervical cancer, radiotherapists, surgeons and urologists should consider the possibility of a high incidence of such serious complications. 


\section{REFERENCES}

1. Tattersall MHN, Larvidhaya V, Vootiprux V. Randomized trial of epirubicin and cisplatin chemotherapy followed by pelvic radiation in locally advanced cervical cancer. Am J Clin Oncol. 13:444-451, 1995

2. Sundfør K, Trope CG, Hogberg T. Radiotherapy and neoadjuvant chemotherapy for cervical carcinoma. A randomized multicenter study of sequential cisplatin and 5-fluorouracil and radiotherapy in advanced cervical carcinoma stage 3B and 4A. Cancer. 77:2371-2378, 1996

3. Leborgne F, Leborgne JH, Doldán R. Induction chemotherapy and radiotherapy of advanced cancer of the cervix: A pilot study and phase III randomized trial. Int J Radiat Oncol Biol Phys. 37:343-350, 1997

4. Guide ALD 30 «Cancer invasif du col utérin »HAS - Service des maladies chroniques et dispositifs d'accompagnement des malades INCa Département des recommandations pour les professionnels de santé Janvier. 2010.
5. The American Cancer Society medical and editorial content team (3rd of january 2020) https://www.cancer.org/cancer/cervicalcancer/treating/radiation.html\#written_by

6. Machida H, Matsuo K, Furusawa A, Kita T, Kitagawa R, Mikami M. Profile of treatmentrelated complications in women with clinical stage IB-IIB cervical cancer: A nationwide cohort study in Japan. PLoS ONE. 2019; 14(1): e0210125. https://doi.org/ 10.1371/journal.pone.0210125.

7. Jérôme Rigaud, Jean-François Hetet, Olivier Bouchot: prise en charge de la cystite radique. Progrès en Urologie.2004, 14, 568-572.

8. Ketata S, Boulaire JL, Al-Ahdab N, Bargain A, Damamme A. Spontaneous intraperitoneal perforation of the bladder: a late complication of radiation therapy for prostate cancer. Clin Genitourin Cancer. 2007 Mar;5(4):287-90.

9. Fujikawa K, Miyamoto T, Ihara Y, Matsui Y, Takeuchi H. High incidence of severe urologic complications following radiotherapy for cervical cancer in Japanese women. Gynecol Oncol. 2001 Jan;80(1):21-3. 